\title{
EFFECTS OF CELLULAR TELEPHONE USE WHILE DRIVING BASED ON OBJECTIVE AND SUBJECTIVE MENTAL WORKLOAD ASSESSMENT
}

\author{
Roberto Abraham Tokunaga, Akihiro Shimojo, \\ Traffic Engineering Division, Road Department \\ Civil Engineering Research Institute of Hokkaido I.A.I. \\ 1-34, Hiragishi 1-3, Toyohira-ku, Sapporo 062-8602 JAPAN \\ Tel: +81-11-841-1738, Fax: 81-11-841-9747, E-mail: roberto@ceri.go.jp \\ Toru Hagiwara, Seiichi Kagaya and Ken-etsu Uchida \\ Transportation \& Traffic Eng. Lab., HOKKAIDO UNIVERSITY \\ North 13, West 8, Kita-ku, Sapporo 060-0813 JAPAN
}

\begin{abstract}
Summary: A series of studies were performed to investigate the effects of cellular telephone use while driving on driver mental workload. In these surveillance studies objective and subjective methods were used to find the driver mental workload behaviour. In the first study, the results indicated that the hands-free system provided less effect on the driver's mental workload than the hand-held system. In the second study, experience in using a cellular telephone while driving had no positive effect on reaction time. The operation task and talking task had little effect on the subjective mental workload of the experienced subjects, but had statistically significant effects on the subjective mental workload of the non-experienced subjects. In the third study, the results of experiment indicated that the telephone tasks increase the mental workload of the drivers as were shown in the second study. The results also indicated that the complex conversation task produced an increase in driver mental workload as compared to the simple conversation task and the other tasks independent of age group.
\end{abstract}

\section{INTRODUCTION}

Alm et al. conducted a number of studies on drivers' behaviour as a function of mobile telephone tasks while driving. These studies were conducted in a VTI driving simulator. They concluded that using a mobile telephone while driving had negative effects on driver behaviour. In 1997, the Japan Safe Driving Centre conducted an experiment on a simulated urban road to investigate cellular telephone use while driving. The results of this investigation showed that hand-held cellular telephone (HHS) use while driving significantly affected the drivers' reaction times, scanning ability and lane-keeping performance. As a result of the findings, in November of 1999 the Japanese Government (e.g., Abe) reformed the traffic law to prohibit cellular telephone use while driving except in some cases. Between 1996 and 1999, we conducted a series of studies (e.g., Tokunaga) to investigate the effects of cellular telephone manipulation and conversation while driving on driver reaction time and subjective mental workload (SMWL). The first study was conducted on a driving simulator developed by the Civil Engineering Research Institute of Hokkaido Development Bureau and the other two studies were conducted on an expressway. In this paper, we will report the results arrived at through these studies. 


\section{CELLULAR TELEPHONE HANDLING (Simulative Experiment)}

The objective of this study was to investigate how the location of a cellular telephone in a vehicle affects driver reaction time and SMWL. A comparative experiment was performed to compare the differences between a HHS and hands-free system (HFS). The experiment was conducted using a driving simulator without motion. An in-screen light was used to simulate a brake light of a leading vehicle. The road type designed on the driving simulator was a straight two-lane expressway and its width was 7 meters. The cellular telephone used in this experiment was placed in two positions. When the test with HFS was performed, the cellular telephone was located on the left side of the dashboard in the driving simulator driven by the subject. When the experiment without HFS was performed, the cellular telephone was located on the front passenger seat. Twenty-four drivers, all men, aged 21 to 51 years old, participated in the experiment.

Major independent variables were the following tasks and the cellular telephone positions (HFS or HHS): receipt task, call task, talk task, radio task, drink task, blinker task and response task. The above tasks, excluding the receipt task were projected on

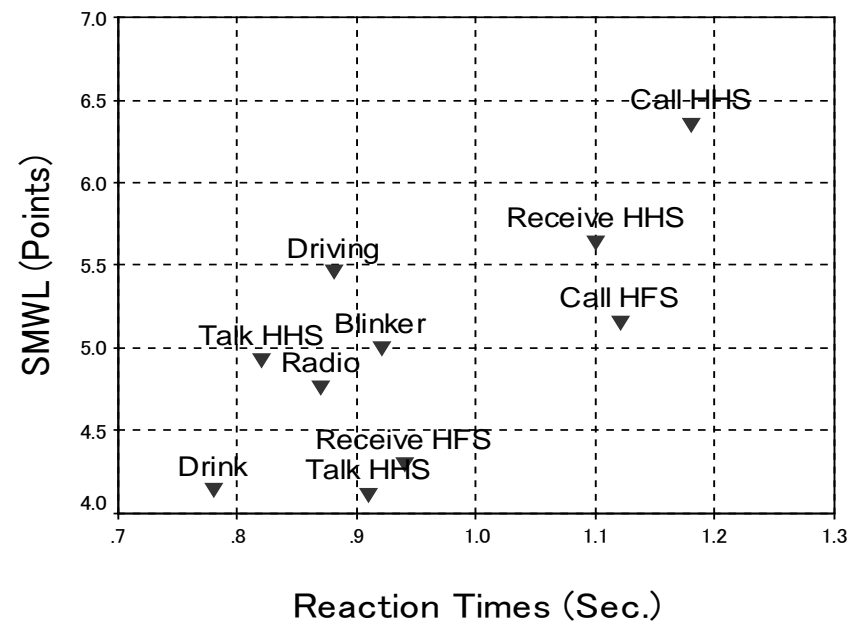

Figure 1 Correlation between dependent variables. the simulator screen like traffic signs. The dependent variables in this study were the driver reaction time and the SMWL. Driver reaction time was defined as the interval from the time when an in-screen light in the driving simulator comes on to the time when the subject presses the brake pedal. A multiple recording data system of the driving simulator measured the drivers' reaction times. The NASA-Task Load Index (NASA-TLX) (e.g., Hart) procedure was used to estimate the drivers' SMWL.

\section{Results}

The call task with HHS indicated the longest drivers’ reaction time (1.18 seconds). Drivers' reaction times in call and receipt tasks were longer than other tasks. T-tests between the response task and others tasks indicated significant differences except with the receipt task with HFS. On the other hand, a T-test between cellular telephone locations indicated no significant differences. Nevertheless, in the experiment we could verify that the drivers missed the stimulus light in 21 opportunities. The major quantity in misses was observed when the subjects performed call and receipt tasks with HHS. In addition, the drivers' reaction times average in the receipt task with HFS was less than the receipt task with HHS ( 0.16 seconds difference).

The highest drivers' SMWL values were seen in the call task with HHS. At the same time, in the telephone tasks the lowest drivers' SMWL values were seen in the talk task with HFS. Tukeys' pair-wise multiple comparisons method was performed to see the differences among task pairs. These results indicated significant differences between call task with HHS and the rest of the tasks. Figure 1 shows the correlation between driver's reaction times and SMWL values. In consequence, both results indicated significant increment when the subjects called or received with HHS. At the same time, talk task and receive task with HFS values were ad eundem to the other comparative tasks. 


\section{CONVERSATION WHILE DRIVING (Field Experiment)}

This study was designed to determine the effects of using a cellular telephone on the reaction time and SMWL of the driver while the driver follows a vehicle at a constant distance. The objectives of this study were to investigate whether using a cellular telephone while driving has negative effects on reaction time and SMWL of a driver under real driving conditions. And also to determine whether a driver who is familiar with the use of a cellular telephone while driving has shorter reaction time and lower SMWL of a driver than these of a driver who is not familiar with the use of a cellular telephone while driving.

Sixteen male civil engineers, 24 to 44 years old, participated in the experiment. Eight of the subjects were familiar with using a cellular telephone while driving, and the other eight subjects had no experience in using a cellular telephone while driving. The experiment was conducted on the Central Expressway of Hokkaido. The total length of the test section was $50.6 \mathrm{~km}$ (round trip). One test section ( $25.3 \mathrm{~km}$ ) was completed in about 20 minutes. Two vehicles were used in the experiment. The subject drove a vehicle following a leading vehicle. A cellular telephone with a HFS was placed on the left side of the dashboard in the experimental vehicle driven by the subject. The dependent

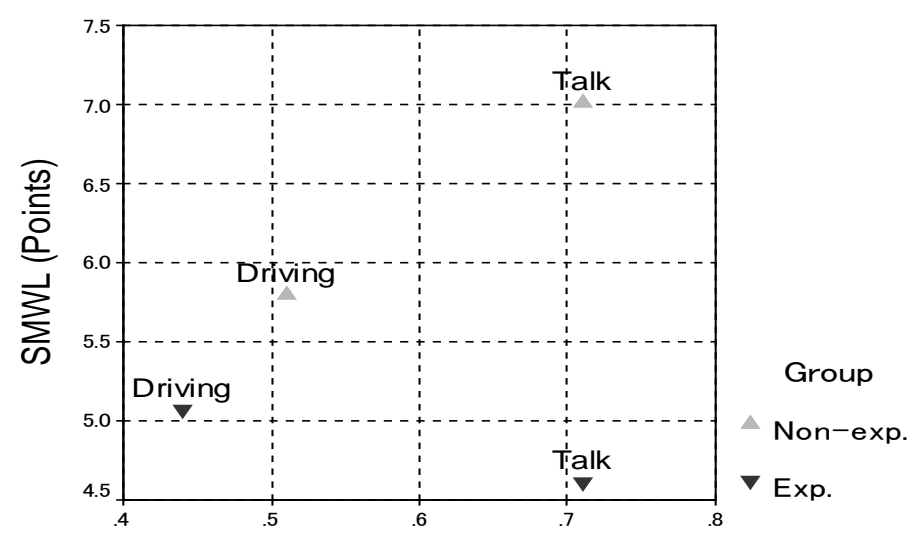

Reaction Times (Sec.)

Figure 2 Correlation between dependent variables in function of experience. variables in this study were the reaction time and the SMWL of a driver. Driver reaction time was defined as the interval from the time when the stoplights of the leading vehicle come on to the time when the subject releases the accelerator pedal. And the NASA-TLX procedure was used to estimate the driver's SMWL. Major independent variables were the following three tasks and experience or inexperience in using a cellular telephone while driving: driving task (car following task), operational task (telephone handling) and talking task.

\section{Results}

The average of driver's reaction time during the driving task was 0.44 seconds in the experienced group and 0.51 seconds in the inexperienced group. The average of reaction time during the talking task was 0.71 seconds in both groups. The reaction times during the driving task showed a narrow distribution in both groups. During the talking task, however, the reaction times in the experienced group were more widely distributed than those in the inexperienced group. A two-way ANOVA was performed to compare the driving task and the talking tasks in each group. The task factor was statistically significant in the both groups, but the group factor was not statistically significant. There was no significant interaction between tasks and groups.

The average values of SMWL of the experienced subjects during the three tasks were similar. The lowest average SMWL was during the talking task. The average values of SMWL of the inexperienced subjects during the operation task and talking task were larger than that of SMWL during the driving task. The dispersion in SMWL of the experienced subjects was larger than that of the inexperienced subjects. A 
two-way ANOVA showed that there were no significant differences between three tasks, and there were no significant differences between the experienced group and inexperienced group. There was statistically significant interaction between tasks and groups. The P-value was 0.032. In case of driving task, a one-way ANOVA between subjects showed that there were no significant effects of experience in using a cellular telephone while driving on SMWL. However, in the case of the operation task and the talking task, a one-way ANOVA between subjects showed that there were significant effects of experience in using a cellular telephone while driving on SMWL. Figure 2 shows the correlation between driver's reaction time and SMWL values in function of their experiences.

\section{CONVERSATION COMPLEXITY (Field Experiment)}

The objective of this study was to investigate whether talking through a cellular telephone while driving has negative effects on driver reaction time and SMWL according to conversation type. At the same time, to determine whether the reaction time and SMWL of an elderly driver using a cellular telephone while driving are better than those of a young driver. The experiment consisted of two types of conversations through a cellular telephone with HFS while driving: the first easy and the other more complex. Thirty-one subjects, 22 to 65 years old, participated in the experiment. All subjects had previous experience using cellular telephones and other communication devices. Nineteen subjects (mean age 23.95 years) were young drivers, and the other twelve subjects (mean age 62.75 years) were elderly drivers. As in the first field experiment the test was conducted on the Central Expressway of Hokkaido. Two vehicles were used in the experiment as in the first field experiment. The subject drove a vehicle following the leading vehicle. A cellular telephone with a HFS was fixed on the left side of the dashboard in the experimental vehicle driven by the subject. Major independent variables were the following four tasks and the drivers' groups (elderly or young). Each subject was required to perform the following four tasks: driving task (car following task), operational task (telephone handling), simple conversation and complex conversation (math problems). As in the others studies, the dependent variables in this investigation were the reaction time and the SMWL of each driver. Drivers' reaction time in this case was defined as the interval from the time when the emergency warning lights of the leading vehicle come on to the time when the subject presses the switch buttons installed on the steering wheel.

\section{Results}

Drivers' reaction time during driving task was less than 0.76 seconds. Drivers' reaction time during the simple conversation task was longer than that of the driving task. In addition, drivers' reaction time during the complex conversation task increased even more than that of the simple conversation task. Thus, a negative effect was found on both groups' reaction times during telephone tasks. Moreover, the 
complexity of conversation directly influenced the increase in drivers' reaction time.

From drivers' reaction times results, a two-way ANOVA was performed to compare the four tasks and groups. There were no significant differences between groups. Tukeys’ pair-wise multiple comparisons method was performed to see the differences among pairs. There were significant increases in drivers' reaction times between the southbound driving task and the simple conversation task, and between the driving task and that for the complex conversation task.

Drivers' SMWL values for the driving task were less than 4.00 points in both groups. Both drivers' SMWL values for the operational task were bigger than that of the driving task respectively. Meanwhile, drivers' SMWL values for the simple conversation task were close to their operational task. Finally, drivers' SMWL values for the complex conversation task increased more than that of the simple conversation. Nevertheless, elderly drivers' SMWL value for the complex conversation task was less than that of the young drivers' SMWL value. Thus, a significant effect was found on both drivers' SMWL for telephone tasks, and the complexity of conversation directly influenced drivers' SMWL. In addition, from drivers' SMWL a two-way ANOVA was performed to compare the six tasks and groups. There were significant differences in the variation pattern between groups $(\mathrm{F}(5,145)=3.254, \mathrm{P}$-value $<0.05)$. Tukeys' pair-wise multiple comparisons method was performed to see the differences among pairs of tasks. There were no significant differences except for the increase in drivers' SMWL for the complex conversation task as compared to the other tasks. Figure 3 shows the correlation between driver's reaction time and SMWL values in function of age groups.

\section{CONCLUSIONS}

The effects of cellular telephone use while driving were investigated from the simulative and field experiments. In them, driver reaction time and SMWL were investigated as a function of cellular telephone location, conversation and conversation complexity. All the studies showed that using a cellular telephone while driving increase the driver reaction time and SMWL.

In the experiment using driving simulator, the results of drivers' reaction times as a function of cellular telephone location indicated no significant differences. However, considering the percentage of stimuli (in-screen light) missed when the drivers performed the receipt and call tasks with HHS, the difference could be significant. The drivers' SMWL values for the telephone tasks with HFS were less than telephone tasks with HHS.

In the first experiment on the expressway, the talking task lead to an increase in the reaction time. Experience in using a cellular telephone while driving had no positive effect on reaction time. The operation task and the talking task had little effect on the SMWL of the experienced subjects, but had statistically significant effects on the SMWL of the inexperienced subjects. Thus, the use of a cellular telephone while a car following drive could increase the risk of an accident even if the driver is experienced in using a cellular telephone while driving. It should be noted however, that the reaction time during the talking task was not so long, indicating that it might be possible to find safe ways of using a cellular telephone while driving.

In the second experiment on the expressway, the drivers' reaction times were longer in both groups while performing the complex conversation task than while performing the simple conversation task. Furthermore, for both age groups the SMWL increased significantly in the complex conversation task as 
compared to the other tasks. However, cellular telephone use as a function of two types of conversation while driving indicated no significant difference between the increment of elderly drivers' reaction times and SMWL and that of young drivers. The performance of elderly drivers' that participated in the field experiment may have been related to extensive annual driving distance and driving experience. In addition, it should be noted that the elderly drivers participating in this experiment were carefully selected for safety according to driving experience and psychophysics capabilities. Therefore, it is necessary to clarify that these results were obtained under limited conditions. In road conditions or situations more complex, it is possible that Drivers' Mental Workload will increase more.

To conclude, the traffic problems originating from cellular telephone use while driving are not only operational questions. They also depend on the information processing capacity of drivers. We think that although it is important to improve the devices, it is also the quantity and quality of information offered to the driver through the cellular telephone as a function of its processing capacity.

\section{REFERENCES}

[1] Abe Hisaaki, Prohibition of Cellular Telephone Use while Driving, Special Edition: The Traffic Law Reform, Traffic Monthly, Tokyo, 1999 (in Japanese).

[2] Alm Hakan et al., Changes in Driver Behavior as a Function of Hands-free Mobile Phones-A Simulator Study, Accident Analysis and Prevention, Vo.26, No.4, pp.441-451, 1994.

[3] Alm Hakan et al., The Effects of a Mobile Telephone Task on Driver Behavior in a Car Following Situation, Accident Analysis and Prevention, Vol.27, No.5, pp.707-715, 1995.

[4] Hart Sandra et al., Development of NASA-TLX: Results of Empirical and Theoretical Research, HUMAN MENTAL WORKLOAD, Elsevier Science Publishers B.V. (Holland), pp.139-183, 1988.

[5] Japan Safe Driving Center et al., Effects of Cellular Telephone Use on Driving Behavior, JSDC Surveillance Study Report, Tokyo, 1997 (in Japanese).

[6] Tokunaga Roberto et al., Effects of Cellular Telephone Manipulation on Driver's Performance, JSAE, Transaction of JSAE, Paper No. 9930432, pp. 127-131, Vol. 30 No. 1, January 1999.

[7] Tokunaga Roberto et al., Effects of Talking through Cellular Telephone on Driving Behavior, Japan Society of Civil Engineers, Infrastructure Planning Review Vol. 16, pp. 895-901, September 1999.

[8] Tokunaga Roberto et al., Effects of Conversation Through a Cellular Telephone While Driving on Driver Reaction Time and Subjective Mental Workload, TRB 79th Annual Meeting, Paper 00-1480, Washington D.C., 2000. 